\title{
Fighting Sleep at Night: Brain Correlates and Vulnerability to Sleep Loss
}

\author{
Micheline Maire, PhD, ${ }^{1,2}$ Carolin Franziska Reichert, $\mathrm{PhD},{ }^{1,2}$ \\ Virginie Gabel, PhD, ${ }^{1,2}$ Antoine U. Viola, PhD, ${ }^{1,2}$ Christophe Phillips, PhD, ${ }^{5}$ \\ Julia Krebs, MD, ${ }^{1,2}$ Klaus Scheffler, PhD, ${ }^{6,7}$ Markus Klarhöfer, PhD, ${ }^{3}$ \\ Werner Strobel, MD, ${ }^{4}$ Christian Cajochen, $\mathrm{PhD},{ }^{1,2 \star}$ and Christina Schmidt, PhD ${ }^{1,2 \star}$
}

Objective: Even though wakefulness at night leads to profound performance deterioration and is regularly experienced by shift workers, its cerebral correlates remain virtually unexplored.

Methods: We assessed brain activity in young healthy adults during a vigilant attention task under high and low sleep pressure during night-time, coinciding with strongest circadian sleep drive. We examined sleep-loss-related attentional vulnerability by considering a PERIOD3 polymorphism presumably impacting on sleep homeostasis.

Results: Our results link higher sleep-loss-related attentional vulnerability to cortical and subcortical deactivation patterns during slow reaction times (i.e., suboptimal vigilant attention). Concomitantly, thalamic regions were progressively less recruited with time-on-task and functionally less connected to task-related and arousal-promoting brain regions in those volunteers showing higher attentional instability in their behavior. The data further suggest that the latter is linked to shifts into a task-inactive default-mode network in between task-relevant stimulus occurrence.

Interpretation: We provide a multifaceted view on cerebral correlates of sleep loss at night and propose that genetic predisposition entails differential cerebral coping mechanisms, potentially compromising adequate performance during night work.

ANN NEUROL 2015;78:235-247

Cleep-loss-related decrements in cognitive performance are most severe during the biological night. ${ }^{1}$ They often originate from a misalignment of circadian and sleep-wake regulatory mechanisms, rendering night work particularly challenging for humans. At the end of the biological night, the endogenous circadian timing system no longer promotes wakefulness, but facilitates sleep with maximal sleep propensity. ${ }^{2}$ The circadian system interacts with a homeostatic process tracking previous sleep-wake history by increasing sleep pressure levels during wakefulness and dissipating during sleep. ${ }^{3}$ Thus, at night, as both the circadian and homeostatic processes promote sleep, ${ }^{2}$ adequate performance levels are compromised by lapses, slower reaction times (RTs), and greater response variance. ${ }^{4}$ Especially in tasks with relatively infrequent stimuli, it is most difficult to sustain appropriate attentional levels over time, whereas phasic adequate recruitment of attentional resources still remains possible. ${ }^{4,5}$ In vigilant attention tasks, the nonoptimal domain (i.e., lapses or slow RTs) is thus more affected by sleep loss and adverse time of day than the optimal domain (i.e., fast responses). ${ }^{6}$ The cerebral basis of attentional processes under sleep deprivation (SD), and in particular how attentional vulnerability to sleep loss becomes evident at

View this article online at wileyonlinelibrary.com. DOI: 10.1002/ana.24434

Received Dec 5, 2014, and in revised form Apr 20, 2015. Accepted for publication May 1, 2015.

Address correspondence to Prof Dr Christian Cajochen, Center for Chronobiology, Psychiatric Hospital of the University of Basel, Wilhelm Klein-Strasse 27, 4012 Basel, Switzerland. E-mail: christian.cajochen@upkbs.ch

*Equal contribution.

From the ${ }^{1}$ Center for Chronobiology, Psychiatric Hospital of the University of Basel, Basel, Switzerland; ${ }^{2}$ Transfaculty Research Platform Molecular and Cognitive Neurosciences, University of Basel, Basel, Switzerland; ${ }^{3}$ Department of Medical Radiology, MR-Physics, University of Basel, Basel, Switzerland; ${ }^{4}$ Respiratory Medicine, Department of Internal Medicine, University Hospital Basel, Basel, Switzerland; ${ }^{5}$ Cyclotron Research Center, Department of Electrical Engineering and Computer Science, University of Liège, Liège, Belgium; ${ }^{6} \mathrm{MRC}$-Department, MPI for Biological Cybernetics, Tübingen, Germany; and ${ }^{7}$ Department of Biomedical Magnetic Resonance, University of Tübingen, Tübingen, Germany. 
the cerebral level at this very critical time (i.e., during the individual biological night), has not yet been investigated. Crucially, up to $92 \%$ of variance in neurobehavioral performance can be explained by systematic, trait-like interindividual variability. ${ }^{7}$ Accordingly, the search for genetic influences of vulnerability to the negative effects of SD was fostered. A variable-number-tandem-repeat (VNTR) polymorphism in the human clock gene PERIOD3 (PER3) was associated with susceptibility to total SD at the physiological and behavioral level, ${ }^{8-11}$ most likely through influence on the sleep homeostatic process. ${ }^{12}$ Notably, so far, PER3-related neurobehavioral modulation was detected under total SD during the night and early morning hours, but not when sleep was merely restricted to a few hours per night. ${ }^{13}$ At the cerebral level, a previous study already revealed that the PER3 polymorphism affected brain activity underlying working memory performance after 24 hours of total SD. ${ }^{14}$ However, like in other SD studies investigating cerebral activity, ${ }^{15}$ data were collected during the biological day following the SD night. Furthermore, sleep-loss-related drifts into taskinactive brain networks might be most likely detected during tasks with relatively low stimulus frequency ${ }^{16}$ at adverse circadian phase during the biological night. Thus, our aim was to investigate the neural bases of vigilant attention during night-time by considering vigilance-specific genetic vulnerability to total SD based on the PER3 polymorphism. We combined a 40-hour SD and a multiple nap protocol (NP) to compare brain activity at the same critical circadian time point under high versus low sleep pressure levels, ${ }^{17}$ with the aim to disentangle sleep homeostatic and circadian influences at the cerebral level. The functional magnetic resonance imaging (fMRI) sessions were scheduled at 21 hours after habitual wake time during each participant's biological night. Based on the link between the PER3 polymorphism and SD vulnerability via the impact on the homeostatic process, ${ }^{12}$ we expected group differences between homozygous short- $\left(P E R 3^{4 / 4}\right)$ and long-allele $\left(P E R 3^{5 / 5}\right)$ carriers to occur mainly under high sleep pressure. We hypothesized that the PER3-related differences will mainly emerge in brain responses associated with slow RTs, given that behavioral ${ }^{6}$ and cerebral $^{18}$ changes in this RT range are considerably prominent after sleep loss. In between the phasic occurrence of stimuli, when attention has to be sustained in the absence of a stimulus, a stronger drift into task-inactive networks ${ }^{19}$ was predicted in $P E R 3^{5 / 5}$ carriers. Notably, vulnerability to total SD comprises many domainspecific aspects (e.g., reference 7). Given that we have recently shown that $P E R 3^{5 / 5}$ carriers are particularly vulnerable to the detrimental effects of total SD in subjective and objective measures of sleepiness and vigilant attention, ${ }^{9}$ we consider this group as the more vulnerable participants here.

\section{Patients and Methods}

\section{Participants}

Thirty-three healthy volunteers participated in the study. One subject was excluded because of fMRI data loss, 2 subjects dropped out of the study, and 2 subjects were not included in this sample because of matching criteria (final $\mathrm{N}=28$; mean age \pm standard deviation: $24.9 \pm 3.3$ years). Details on recruitment and exclusion criteria can be found in a previous work. ${ }^{9}$ Fourteen ( 7 males, 7 females, 1 left-handed) were homozygous carriers of the short repeat allele $\left(P E R 3^{4 / 4}\right)$ and 14 (5 males, 9 females) homozygous carriers of the long repeat allele $\left(P E R 3^{5 / 5}\right.$, all right-handed). The genotyping procedure has been described in previous reports. ${ }^{9,20}$ Women without contraceptives (2 of 16) participated during the luteal phase of their menstrual cycle. The groups did not significantly differ in terms of gender ratio, age, body mass index, bed times during study weekends, and questionnaire scores (Table 1). The local ethics committee (Ethikkomission beider Basel, EKBB, Switzerland) approved the study, and all procedures conformed to the standards of the declaration of Helsinki. Written informed consent was given.

\section{Procedure}

The study procedure is illustrated in Figure 1. Behavioral, melatonin, and electrophysiological data of this design characterizing our sample according to genotype have been published before, where details on the study procedure can be found. Here, the most important aspects will be reproduced. Each volunteer completed two study blocks (56-hour duration each) in the laboratory, in a pseudo-randomized, balanced, crossover order. We implemented stringently controlled conditions, which are semirecumbent posture in bed during wakefulness, regularly scheduled light meals, dim light $(<8$ lux $)$ during scheduled wakefulness, and zero lux with supine body posture during scheduled sleep episodes (i.e., naps), as well as no time-of-day indication. Participants were continuously monitored by electroencephalography (EEG) except during fMRI acquisition. Both protocols started with a baseline night ( 8 hours time in bed at respective habitual bedtimes, which were held regular during the week before study blocks). In the SD, participants were awake for 40 hours after habitual wake-up time. In the NP, they underwent 10 alternating cycles of 160 minutes of scheduled wakefulness and 80 minutes of scheduled sleep (i.e., naps). Both blocks ended with a recovery night (minimum 8-hour time in bed at usual bedtimes). The combination of the two protocols allows for investigating a continuous rise in homeostatic sleep pressure on one hand, whereas on the other hand, bymultiple naps, sleep pressure remains at relatively low levels (see also previous works ${ }^{9,17,21}$ ).

Here, we focus on the functional imaging data acquired during the night, that is, 21 hours after wake up (approximately 4 AM for a 7 AM wake-up time). The dim-light melatonin offset (DLMOff), indicative for the passage into the biological day, occurred, on average, at $23.55 \pm 1.3$ hours after wakeup in the NP protocol and $23.47 \pm 1.4$ hours in the SD protocol. DLMOff was determined at the $50 \%$ level of the maximal 
TABLE 1. Means ( \pm Standard Deviation) of Demographic Data and Questionnaire Scores by PER3 Genotype

\begin{tabular}{|c|c|c|c|}
\hline & $P E R 3^{4 / 4}$ & PER3 $^{5 / 5}$ & $p$ \\
\hline $\mathrm{N}[\mathrm{M}, \mathrm{F}]$ & $14[7,7]$ & $14[5,9]$ & 0.44 \\
\hline Years of age & $24.3(3.0)$ & $25.6(3.6)$ & 0.31 \\
\hline BMI $\left[\mathrm{kg} / \mathrm{m}^{2}\right]$ & $21.9(1.9)$ & $22.7(2.8)$ & 0.38 \\
\hline Wake time [clock time] & $07: 10(61 \mathrm{~min})$ & 07:10 (44 min) & 1.00 \\
\hline Sleep time [clock time] & $23: 10(61 \mathrm{~min})$ & $23: 10$ (44 min) & 0.97 \\
\hline PSQI & $3.3(1.1)$ & $3.0(1.4)$ & 0.54 \\
\hline ESS & $3.6(1.9)$ & $4.3(2.7)$ & 0.47 \\
\hline MEQ & $58.1(9.5)$ & $53.5(10.2)$ & 0.23 \\
\hline MCTQ Sleep duration [hours] & $7.8(0.7)$ & $7.9(1.0)$ & 0.69 \\
\hline MCTQ MSFsc & $4.3(1.0)$ & $4.4(1.3)$ & 0.81 \\
\hline MCTQ MSFsac & $7.5(2.7)$ & $7.2(2.5)$ & 0.54 \\
\hline BDI-II & $2.6(2.5)$ & $1.3(1.9)$ & 0.13 \\
\hline \multicolumn{4}{|c|}{$\begin{array}{l}p \text { values were derived from } X^{2} \text { (gender) and } t \text { tests (all other). } \\
\text { M, male; F, female; BMI = body mass index; PSQI = Pittsburgh Sleep Quality Index }{ }^{49} \text {; ESS }=\text { Epworth Sleepiness Scale }^{50} \text {; } \\
\text { MEQ = Morningness-Eveningness Questionnaire } e^{51} ; M C T Q=\text { Munich Chronotype Questionnaire }{ }^{52} ; \text { MSFsc= Midsleep free days } \\
\text { sleep corrected; MSFsac = Midsleep free days sleep and age corrected; BDI = Beck Depression Inventory-II }{ }^{53} \text {. Wake and sleep } \\
\text { times refer to baseline and recovery nights during the study. }\end{array}$} \\
\hline
\end{tabular}

melatonin secretion. Figure 1 illustrates the overlap of the acquisition point with highest levels of physiological sleepiness (slow eye movements and unintentional sleep episodes assessed and analyzed as described in a previous work ${ }^{9}$ ), especially under high sleep pressure (Fig 1A) and peak levels of melatonin secretion during our study.

\section{Behavior}

PSYCHOMOTOR VIGILANCE TASK. We assessed vigilant attention during both the NP and SD protocol with a psychomotor vigilance task (PVT) of 10 minutes duration. ${ }^{22}$ The original PVT design ${ }^{22}$ was modified to suit fMRI admission. A white fixation cross was presented on a black screen, and at random intervals (2-10 seconds) a millisecond counter started (clock event). Participants were instructed to press a button to stop the counter as fast as possible with their dominant hand. Null events, where the clock event is replaced by the fixation cross, were included at random in the task $(25 \%$ of the trials, 2-10 seconds duration). Feedback of RT performance was displayed for 1 second after the response. RTs $>500 \mathrm{~ms}$ were classified as lapses, which were transformed by $\sqrt{ } \mathrm{x}+\sqrt{\mathrm{x}}+1^{6}$. Timeon-task (ToT) was considered by comparing RTs or number of lapses during the first vs. the last 3 minutes on the task (as previously published ${ }^{20}$ ). Errors of commission (i.e., random or anticipatory button presses) were not registered.

SUBJECTIVE SLEEPINESS. Participants regularly indicated their subjective sleepiness levels on the Karolinska Sleepiness Scale $\left(\mathrm{KSS}^{23}\right)$. Here, we report an average value (mean sam- pling time \pm standard deviation: 03:22 $\mathrm{AM} \pm 10$ minutes) of three night-time samplings during SD (approximate times: 02:30, 03:30, and 04:00 AM; similar for the two genotypes, $p>0.05)$ preceding the fMRI acquisition within the biological night.

BEHAVIORAL DATA ANALYSIS. Group analyses of the behavioral data (i.e., PVT performance and subjective sleepiness) were performed with the statistical package SAS (version 9.3; SAS Institute Inc., Cary, NC). We used $t$ tests or mixedmodel repeated measures analysis of variance (PROC MIXED), where appropriate, and $p$ values were based on KenwardRoger's corrected degrees of freedom. ${ }^{24}$ Contrasts were assessed with the LSMEANS statement. The factors genotype (PER3 $3^{5 / 5}$ vs. PER3 $\left.3^{4 / 4}\right)$, condition (NP vs. SD), and ToT (last vs. first part) were used. For the PVT, RTs were classified as follows for each participant in each session: RTs lower than the 25th percentile (fast RTs); RTs higher than the 75th percentile (slow RTs); and RTs in the range between the 25th and 75th percentile (intermediate RT), and lapses (RTs $>500 \mathrm{~ms}$ ). Unlike our and other previous studies, ${ }^{6,9,18,20}$ we chose this RT range classification to enhance the number of events for fMRI analysis (mean no. of events \pm standard deviation: $14.8 \pm 3.7$ ). Regression analyses were calculated with STATISTICA 9 (StatSoft Inc., Tulsa, OK).

\section{fMRI}

DATA ACQUISITION. A 3T MR Scanner (MAGNETOM Verio; Siemens Healthcare, Malvern, PA) with a standard 


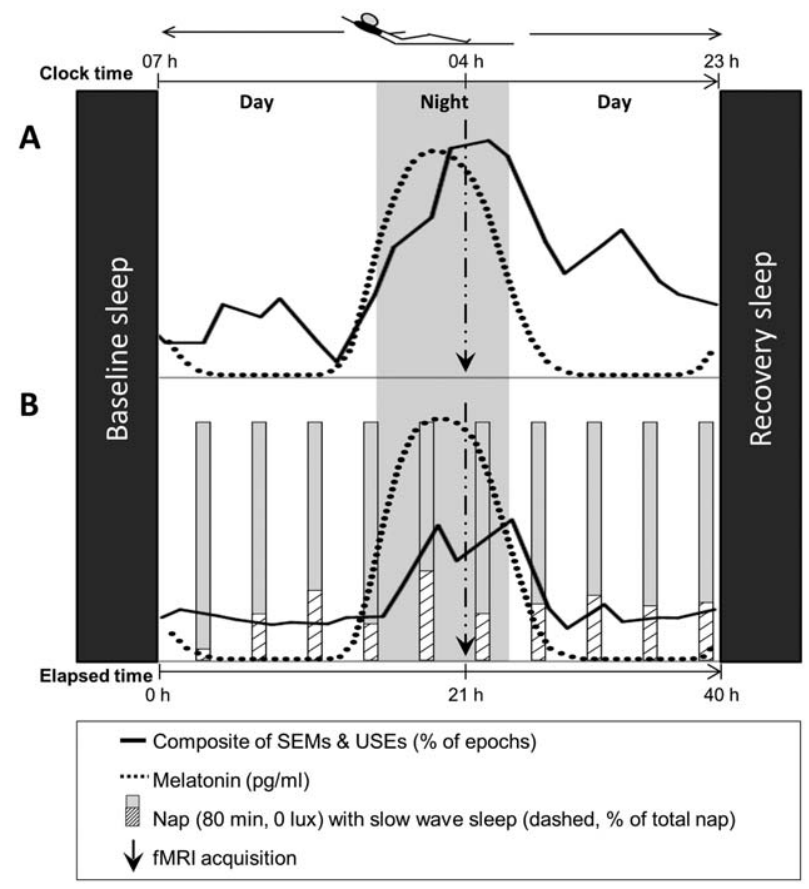

FIGURE 1: Schematic illustration of the laboratory part. (A) 40-hour sleep deprivation (SD). (B) 40-hour multiple nap protocol (ten 80/160-minute sleep/wake cycles). Clock time indication is relative to a $7 \mathrm{AM}$ wake time. In (A) and (B), the solid line shows the 40-hour time course of physiological sleepiness represented by the averaged amount of slow eye movements (SEMs) and unintentional sleep episodes (USEs; mean \% of 20 -second epochs containing slow eye movements or any sleep stage, plotted from zero, $\mathrm{SD}_{\max } \mathbf{2 2 . 7 \%}$, $\mathrm{NP}_{\max } 13.5 \% ; \mathrm{N}=28$; time binned and analyzed as described previously $\left.{ }^{9}\right)$. Dotted lines represent the 40-hour time course of melatonin secretion $(\mathrm{pg} / \mathrm{ml}$, plotted from zero, maximal levels: SD $14.5 \mathrm{pg} / \mathrm{ml}$, NP $13.8 \mathrm{pg} / \mathrm{ml}$; $\mathrm{N}=28$, hourly intervals modeled as described previously ${ }^{9}$; the gray bars in (B) depict the 10 naps, in dashed the respective percentage of slow-wave sleep (see our previous publication 9 for details on analysis of these data). The course of slow-wave sleep illustrates presumable sleep pressure dissipation depending on time of nap. The time of functional magnetic resonance imaging (fmri) data acquisition is marked with the arrow and coincides with peaking melatonin secretion and highest sleepiness levels in SD.

12-channel head coil was used to acquire fMRI time series. Multislice T2*-weighted fMRI images were obtained with a gradient echo-planar sequence using axial slice orientation (32 slices; voxel size: $3 \times 3 \times 3 \mathrm{~mm}^{3}$ with $0.75 \mathrm{~mm}$ interslice gap; matrix size $76 \times 76 \times 32$; repetition time $[\mathrm{TR}]=2,200 \mathrm{~ms}$; echo time $[\mathrm{TE}]=32 \mathrm{~ms}$; flip angle $[\mathrm{FA}]=82^{\circ}$ ). Structural T1weighted images were acquired for anatomical reference with a magnetization-prepared rapid gradient echo sequence $\left(\mathrm{TR}=2,000 \mathrm{~ms} ; \quad \mathrm{TE}=3.37 \mathrm{~ms} ; \quad \mathrm{FA}=8^{\circ} ;\right.$ field of view $=25.6 \mathrm{~cm}$; matrix size $=256 \times 256 \times 176$; voxel size $=1 \times 1 \times 1 \mathrm{~mm}^{3}$ ). One hundred seventy-six contiguous axial slices covering the entire brain were assessed in sagittal direction.

\section{Data Analysis}

GENERAL APPROACH. Data were analyzed with SPM8 (http://www.fil.ion.ucl.ac.uk) implemented in MATLAB 2012 (MathWorks, Natick, MA). Scans were realigned with iterative rigid body transformations, corrected for head motion and spatially normalized (standard SPM8 parameters). In a two-step analysis, we took into account the intra- and interindividual variance, respectively. Brain responses were modeled for each subject at each voxel using a general linear model (GLM). Our model included four regressors for each condition: events associated with RTs lower than the 25th percentile (fast RTs); events associated with RTs higher than the 75th percentile (slow RTs); events linked to the RT range between the 25th and 75th percentile (intermediate RTs) as well as lapses (RTs $>500 \mathrm{~ms}$ ). For each trial type, we additionally added a time modulation regressor (first-order polynomial) to account for ToT effects. Every event of each trial type was modeled as a function representing its onset (i.e., at the time of presentation of stimulus). The ensuing vector was convolved with the canonical hemodynamic response function (HRF) and used as a regressor in the individual design matrix. The main effect of task performance during the different test sessions was estimated with linear contrasts. We focused on the RT range encompassing the mean of the slowest $25 \%$ of RTs. This RT range is very sensitive to increased sleep pressure ${ }^{6,18,25}$ and its neural basis has been previously described. ${ }^{18}$ Please note that unlike other authors, ${ }^{6,18}$ we separated lapses (RT > $500 \mathrm{~ms}$ ) from the slowest RTs (thus, maximal duration of a slow RT was $500 \mathrm{~ms}$ ) in our study. This ensures that we only investigate brain activity when participants were awake and able to respond. Furthermore, at the between-subject level, we separately included subjective sleepiness as well as the mean speed difference between each slowest and its subsequent RT as covariates to investigate the relation to cerebral activity during SD.

Statistical inferences were performed at a threshold of $p=0.05$ after correction for multiple comparison over the entire brain (family-wise error correction) or over small spherical volumes (radius, $10 \mathrm{~mm}$ ) around regions of interest (ROIs) at locations a priori derived from the literature (small volume correction, svc). These ROIs were based on two studies that previously investigated the neural correlates of the PVT, ${ }^{18,26}$ one study reporting cerebral patterns after SD in the two PER3 genotypes, ${ }^{14}$ and three studies reporting the effects of SD on different attentional components. ${ }^{27-29}$ For task-inactive network regions, we identified ROIs based on references previously reporting default mode activity. ${ }^{19,30-33}$

FUNCTIONAL CONNECTIVITY ANALYSIS. To assess functional connectivity between ROIs, we conducted a psychophysiological interaction analysis ${ }^{34,35}$ implemented in SPM8. Based on an a posteriori hypothesis established on the GLM analysis described above, we defined a seed ROI within a region in the brainstem compatible with a part of the reticular formation (see Table S1 and Fig 2), activity in a similar area was previously described to be negatively correlated with deep sleep. ${ }^{36}$ In this 


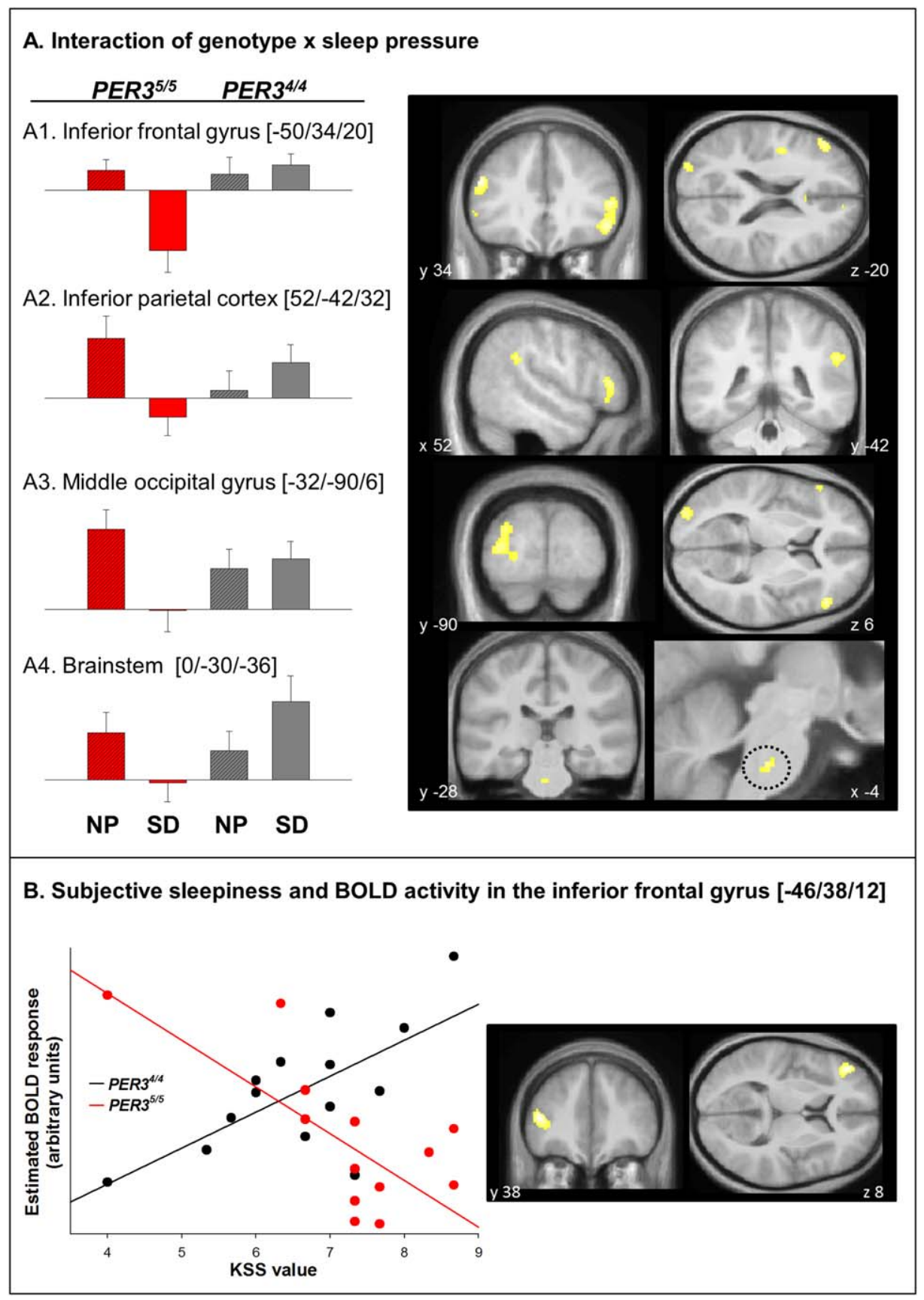

FIGURE 2: BOLD activity during SD vs. NP, PER $3^{44}$ vs. $P E R 3^{55}$ and relation to subjective sleepiness. (A1-A4) Selected brain areas during slowest reaction times showing a significant condition $\times$ genotype interaction (PER $3^{44}$ vs. PER ${ }^{55}, S D$ vs. NP) see also Table 3 and S1). Bar plots display parameter estimates for BOLD activity in regions A1-A4 by genotype and condition on the left. On the right, the corresponding activity overlay on study population mean structural image show higher activations under high sleep pressure (sleep deprivation; SD) compared to low sleep pressure (nap protocol; NP), depending on genotype (display at $p=0.001$ uncorrected). The observed pattern in regions A1-A4 is representative for brain areas listed in Table 3 and S1: The PER ${ }^{55}$ group consistently shows a decrease in activation in SD compared to NP, whereas the PER $3^{44}$ group shows the opposite pattern. Red bars: PER $3^{55}$; black bars: PER $3^{44}$ carriers; dashed bars: NP; filled bars: SD. (B) Left panel: Regression analysis of the relation between estimated BOLD responses during slowest RTs in the left inferior frontal gyrus and the Karolinska Sleepiness Scale (KSS) values (the higher, the sleepier) during the biological night preceding $\mathrm{fMRI}$ acquisition $\left(R^{2}=0.56\right.$; $p<0.001 ; n=28$ ). Right panel: Display of the brain area showing a significant covariance pattern with subjective sleepiness depending on genotype: left inferior frontal gyrus [peak voxel: $-46 / 38 / 12$ ]; $p_{\text {corrected }}<0.000 ; Z=3.8$, display at $p=0.001$ uncorrected). Exclusion of outliers visible on left panel did not change the observed pattern. Activity overlay on study population mean structural image on the right. BOLD =blood-oxygen-level dependent; $f M R I=$ functional magnetic resonance imaging. 
seed region, the first eigenvariate of the blood-oxygen-leveldependent (BOLD) time series was extracted from voxels within a $6-\mathrm{mm}$ radius sphere around the peak voxel (1 male $P E R 3^{5 / 5}$ carrier was excluded from this analysis because the individual activation mask did not cover this brain area, i.e., no data available).

BOLD ACTIVITY BEFORE STIMULUS APPEARANCE. To assess brain activity immediately before stimulus appearance, we further added finite-impulse-response $\left(\mathrm{FIR}^{37}\right)$ regressors for each RT range and its time modulation (fastest, slowest, and intermediate RTs) to our initial model described above. With FIR analysis, the BOLD signal is modeled without assumption of its shape by using successive boxcar functions (miniboxcars or "sticks"). Here, in order to capture BOLD signal right before stimulus appearance, we added one single prestimulus stick with a duration of 2.2 seconds (i.e., one TR). Although stimulus appearance was not synchronized to the TR, with this approach, it is assured that we are capturing prestimulus BOLD activity, given that the HRF associated with the stimulus per se has a 4- to 6-second lag.

\section{Results}

Time Course of Sleepiness and Vigilant Attention Over the Entire $\mathbf{4 0}$ Hours

The typically observed ${ }^{17}$ circadian- and sleep-pressurelevel-dependent time course for subjective and physiological sleepiness was detected throughout the 40-hour SD and multiple nap protocol, as published in a previous work. ${ }^{9}$ As depicted in Figure 1, slow rolling eye movements and unintentional sleep episodes as a physiological correlate for sleep drive increased when passing into the biological night. Maximal levels were reached toward the end of the night to the beginning of the biological day, stabilizing on the second day under SD and decreasing again under NP (related statistics are found in a previous work ${ }^{9}$ ). A similar pattern was observed for lapses (RT $>500 \mathrm{~ms}$ ) during psychomotor vigilance task (PVT) performance (see our previous publication' for details). Importantly, over the 40 hours of SD, PER3 ${ }^{5 / 5}$ participants felt sleepier and had more slow eye movements and unintentional sleep episodes as well as attentional lapses under SD, compared to NP. ${ }^{9}$ According to these findings, clearly revealing different responses to sleep loss in sleepiness and vigilant attention measures, we considered our $P E R 3^{5 / 5}$ carriers as the more SD-vulnerable group hereafter.

\section{Subjective Sleepiness and Vigilant Attention During Night-time}

Participants tended to be subjectively sleepier (assessed with the $\mathrm{KSS}^{23} ; t_{54}=-1.74 ; p=0.08$ ) and had significantly more lapses and longer RTs in the slow domain (25\% slowest RTs) during the night under SD, com- pared to NP. ToT effects in the night session were similar in both conditions for the $25 \%$ slowest RTs and lapses; results are listed in Table 2 . Between genotypes, subjective sleepiness levels were similar during night-time $\left(t_{26}=-1.49 ; \quad p=0.15 ;\right.$ mean \pm standard error [SE]: $P_{E R 3^{4 / 4}} 6.6 \pm 0.3 \quad$ PER3 $\left.^{5 / 5} 7.3 \pm 0.3\right)$. However, the $P E R 3^{5 / 5}$ carriers produced significantly more attentional lapses (number of RTs $>500$ ) than the $P E R 3^{4 / 4}$ carriers $\left(p=0.03 ; \quad\right.$ mean $\pm \mathrm{SE}: \quad P E R 3^{4 / 4} \quad 4.6 \pm 0.7 \quad$ PER35/5 $7.2 \pm 1.1)$ at this time window under $\mathrm{SD}$, irrespective of ToT. RTs did not differ in the slow RT range between genotypes (mean $\mathrm{RT} \pm \mathrm{SE}$ during SD: $P E R 3^{4 / 4}$ 403.9 \pm 3.7, PER35/5 409.1 \pm 4.6 ; mean RT $\pm \mathrm{SE}$ during NP: PER3 $3^{4 / 4} 373.9 \pm 4.1, \quad$ PER35/5 $381.8 \pm 3.9$; mean number of slow responses: $P E R 3^{4 / 4}$ SD 14.9; NP 16.4, $P E R 3^{5 / 5}$ SD 11.9; NP 16.1), nor was the interaction with condition (i.e., SD and NP) significant. ToT affected $P E R 3^{5 / 5}$ carriers similarly for the slow RT range (slower in the last test part).

\section{fMRI Data}

SLEEP PRESSURE MODULATES NIGHT-TIME BRAIN RESPONSES UNDERLYING SLOW REACTION TIMES. Under high sleep pressure, a bilateral thalamic region $\left([0,10,6], Z=3.55, p_{\text {svc }}{ }^{26}=0.0002\right)$ as well as the putamen $\left([22,10,12], Z=3.78, p_{s v c}{ }^{26}=0.0001\right)$ showed higher BOLD activity compared to NP (SD $>N P)$. During NP, several cortical regions, that are, the left cuneus ([6, 94, 20], $\left.Z=3.49, p_{s v c}{ }^{28}=0.0002\right)$, right middle temporal gyrus $\left([64,30,-4], Z=3.91, p_{s v c}{ }^{14}<0.0000\right)$, as well as insular regions $\left([42,18,8], Z=3.2, p_{s v c}{ }^{26}=0.0007\right)$ were more active than during $\mathrm{SD}(\mathrm{NP}>\mathrm{SD})$.

\section{GENETIC VULNERABILITY TO SLEEP LOSS ACUTELY AFFECTS SLEEP-PRESSURE-DEPENDENT BOLD ACTIVITY AND FUNCTIONAL CONNECTIVITY TO AROUSAL-PROMOTING SUBCORTICAL REGIONS DURING NIGHT-TIME VIGILANCE. The PER3 polymor-} phism significantly modulated brain responses to the task according to sleep pressure level (interaction condition $\times$ genotype; see Fig 2; Table S1, significant interaction terms are marked by an asterisk). Generally, $P E R 3^{4 / 4}$ individuals had increased activation during SD compared to NP in multiple attention-related cortical and subcortical brain areas, whereas the $P E R 3^{5 / 5}$ carriers showed the opposite pattern (see Fig 2 for selected regions with corresponding parameter estimates, Table S1 details the corresponding post-hoc contrasts). More precisely, higher BOLD activity under SD was observed for $P E R 3^{4 / 4}$ compared to $P E R 3^{5 / 5}$ carriers (SD 44>55) in frontal, temporal, and parietal areas as well as in a brainstem region. No regions were activated more in $P E R 3^{5 / 5}$ than $P E R 3^{4 / 4}$ carriers during $\mathrm{SD}$ 


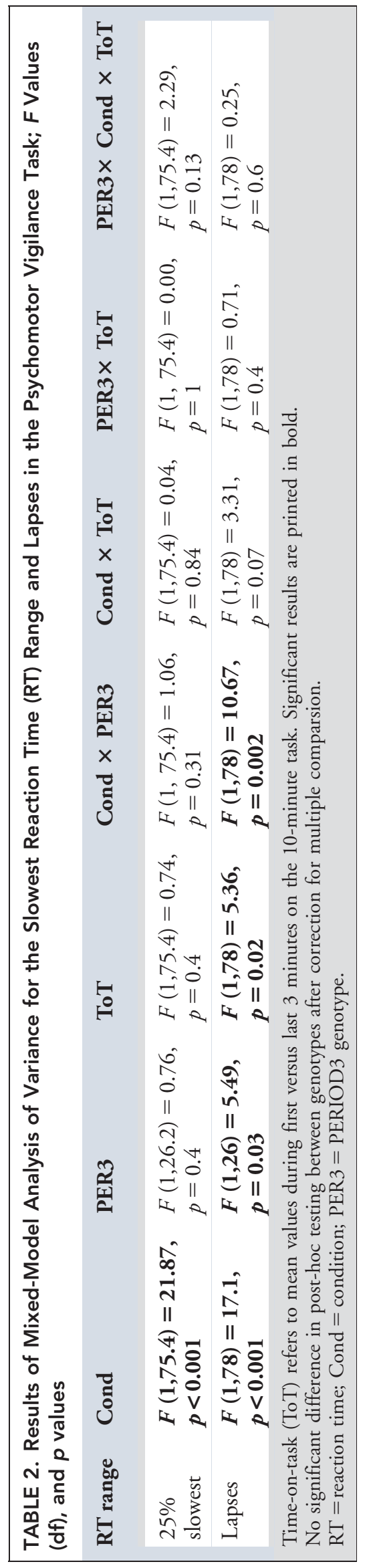

(SD 55>44). Furthermore, separate analyses by genotype revealed that the $P E R 3^{4 / 4}$ group showed BOLD activity increases in SD compared to NP in a series of cortical and thalamic structures as well as areas of the basal ganglia and the cerebellum (44 SD $>\mathrm{NP}$ ). The PER3 ${ }^{5 / 5}$ group yet showed the opposite pattern: None of the areas showed higher activations during SD compared to NP (55 SD $>\mathrm{NP}$ ), but a series of areas were more activated during NP ( $55 \mathrm{NP}>\mathrm{SD}$ ), that is, frontal, temporal, parietal, and several occipital areas as well as a brainstem area compatible with the location of the reticular formation (midline pons). A functional connectivity analysis revealed that under sleep loss, the latter region was more connected to cortical and thalamic structures and a more superior located brainstem region (Table 3) in $P E R 3^{4 / 4}$ compared to $P E R 3^{5 / 5}$ carriers.

Our data indicated that the more resistant individuals $\left(P E R 3^{4 / 4}\right)$ responded to sleep loss by increasing taskrelated cortical BOLD activity and by additionally recruiting thalamic resources. Activity in a subset of these areas also covaried with speed during the subsequent RTs (Table S1, common activity identified by inclusive masking). In these areas, the higher the BOLD activity during a slowest RT, the faster the subsequent RT. The more vulnerable genotype $\left(P E R 3^{5 / 5}\right)$, however, mainly responded by activity decreases and reduced functional connectivity between brainstem, thalamic, and taskrelated cortical regions.

BRAIN RESPONSES UNDERLYING TIME-ON-TASK MODULATION: GREATER STATE INSTABILITY BY DECREASED THALAMIC RECRUITMENT? According to the state-instability hypothesis, ${ }^{4,5}$ SD strongly affects the ability to maintain appropriate attentional levels continuously over time. In a previous report, ${ }^{20}$ we observed that $P E R 3^{5 / 5}$ carriers presented higher response variability and stronger ToT decrements (lapses) in vigilant attention under sleep loss. We detected that the time course of BOLD activity throughout the 10-minute PVT differed between genotypes in the anterior and posterior thalamus as well as the anterior cingulate cortex (Table 4; Fig $3 \mathrm{~A}$ ) under $\mathrm{SD}$ for the slow RT range. $P E R 3^{5 / 5}$ carriers showed activity decreases in these brain areas over the course of the task $(p<0.05)$, whereas activation in $P E R 3^{4 / 4}$ carriers remained stable $(p>0.05$; Fig 3B schematically depicts the course of thalamic brain activity for two representative subjects of each genotype). In fact, the group presenting higher state instability at the behavioral level $^{20}$ was hallmarked at the cerebral level by reduced task-related cingulate and thalamic recruitment with increasing ToT. 


\begin{tabular}{|c|c|c|c|c|c|c|c|}
\hline Brain Area & Side & Z Score & $p_{s v c}$ & $\mathbf{x}$ & $\mathbf{y}$ & $\mathbf{z}$ & Ref. \\
\hline \multicolumn{8}{|c|}{ Brain areas with greater connectivity to the seed region in $P E R 3^{44}$ compared to $P E R 3^{55}$ carriers $(S D 44>55)$} \\
\hline IFG & $\mathrm{L}$ & 3.12 & 0.0009 & -44 & 28 & -4 & 14 \\
\hline \multirow[t]{4}{*}{ Thalamus } & $\mathrm{R}$ & 3.70 & 0.0001 & 26 & -30 & -4 & 14 \\
\hline & $\mathrm{R}$ & 3.40 & 0.0003 & 20 & -18 & -10 & 14 \\
\hline & $\mathrm{R}$ & 3.23 & 0.0006 & 18 & -14 & -10 & 14 \\
\hline & $\mathrm{B}$ & 3.97 & $<0.0001$ & 0 & -8 & 2 & 26 \\
\hline \multirow[t]{2}{*}{ Thalamus/pineal gland } & $\mathrm{B}$ & 3.84 & 0.0001 & -6 & -32 & 2 & 18 \\
\hline & $\mathrm{B}$ & 3.50 & 0.0002 & 2 & -32 & 6 & 18 \\
\hline Brainstem & $\mathrm{L}$ & 3.28 & 0.0005 & -10 & -22 & -16 & 26 \\
\hline \multicolumn{8}{|c|}{ Brain areas with greater connectivity to the seed region in $P E R 3^{55}$ compared to $P E R 3^{44}$ carriers (SD 55 $\left.>44\right)$} \\
\hline \multicolumn{8}{|c|}{ n.s. at $p=0.001$ uncorrected level } \\
\hline \multicolumn{8}{|c|}{$\begin{array}{l}\text { Coordinates }(\mathrm{x}, \mathrm{y}, \mathrm{z}) \text { are expressed in } \mathrm{mm} \text { in the Montreal Neurological Institute }(\mathrm{MNI}) \text { space. } p_{\text {svc }} \text { : } p \text { value after correction for } \\
\text { multiple comparisons over small volumes of interest taken from the literature. } \\
\mathrm{SD}=\text { sleep deprivation; Ref. = references for coordinates; } \mathrm{IFG}=\text { inferior frontal gyrus; } \mathrm{R}=\text { right; } \mathrm{L}=\text { left; } \mathrm{B}=\text { bilateral; n.s. }=\text { no } \\
\text { significant. }\end{array}$} \\
\hline
\end{tabular}

SUBJECTIVE SLEEPINESS MODULATES BRAIN RESPONSES UNDERLYING SLOW RTS DEPENDING ON VULNERABILITY TO SLEEP LOSS. Depending on genotype when sleep deprived, BOLD activity associated to slow RTs was significantly related to subjective sleepiness in the left inferior frontal gyrus (IFG; peak coordinates: -46 ,
38, 12; $\left.\mathrm{Z}=3.8, \mathrm{p}_{\mathrm{svc}}<0.001\right): P E R 3^{5 / 5}$ carriers showed a negative relation (the sleepier they felt, the less they recruited this region), whereas in $P E R 3^{4 / 4}$ carriers, the relation was positive (the sleepier they felt, the more they activated this region; Fig 2E).

TABLE 4. Brain Activity Modulated by Time-on-Task During Sleep Deprivation

\begin{tabular}{llllllll} 
Brain Area & Side & Z Score & $\boldsymbol{p}_{\boldsymbol{s v c}}$ & $\mathbf{x}$ & $\mathbf{y}$ & $\mathbf{z}$ & $\mathbf{R e f}$ \\
\hline $\mathbf{4 4}>\mathbf{5 5}$ & & & & & & \\
\hline Anterior cingulate cortex & $\mathrm{B}$ & 3.39 & 0.0004 & 0 & 32 & 14 & 18 \\
& $\mathrm{R}$ & 3.23 & 0.0006 & 4 & 26 & 22 & 18 \\
\hline Insula & $\mathrm{L}$ & 3.33 & 0.0004 & -30 & -26 & 14 & 27 \\
\hline Midbrain/CMN & $\mathrm{R}$ & 3.41 & 0.0003 & 8 & -22 & -8 & 27 \\
\hline Midbrain/N. ruber & $\mathrm{R}$ & 3.29 & 0.0005 & 10 & -18 & -6 & 26 \\
\hline Midbrain/MDN & $\mathrm{L}$ & 3.32 & 0.0004 & -8 & -16 & -4 & 26 \\
\hline Midbrain/N. ruber & $\mathrm{L}$ & 3.29 & 0.0005 & -6 & -20 & -6 & 14
\end{tabular}

$55>44$

n.s. at $p=0.001$ uncorrected level

Coordinates $(\mathrm{x}, \mathrm{y}, \mathrm{z})$ are expressed in $\mathrm{mm}$ in the Montreal Neurological Institute (MNI) space. $p_{s v c}: p$ value after correction for multiple comparisons over small volumes of interest taken from the literature.

Ref. = references for coordinates; $\mathrm{R}=$ right; $\mathrm{L}=$ left; $\mathrm{B}=$ bilateral; $\mathrm{N}$. = nucleus; $\mathrm{CMN}=$ central medial nucleus of the thalamus; $\mathrm{MDN}=$ medial dorsal nucleus of the thalamus. 
A1

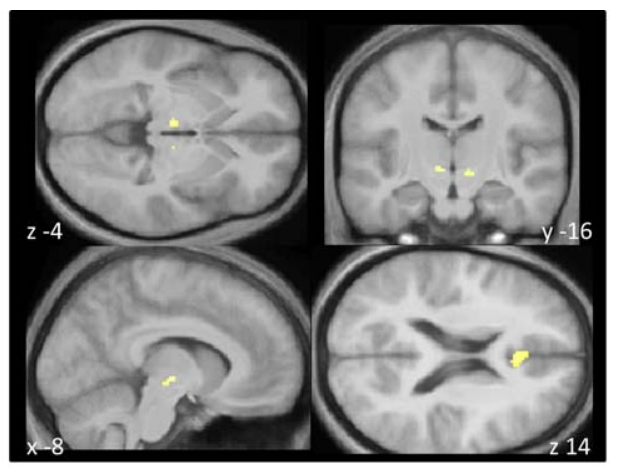

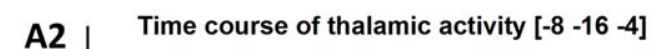

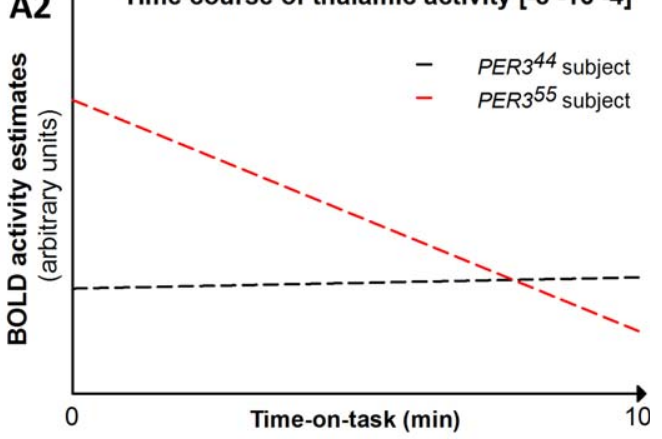

B

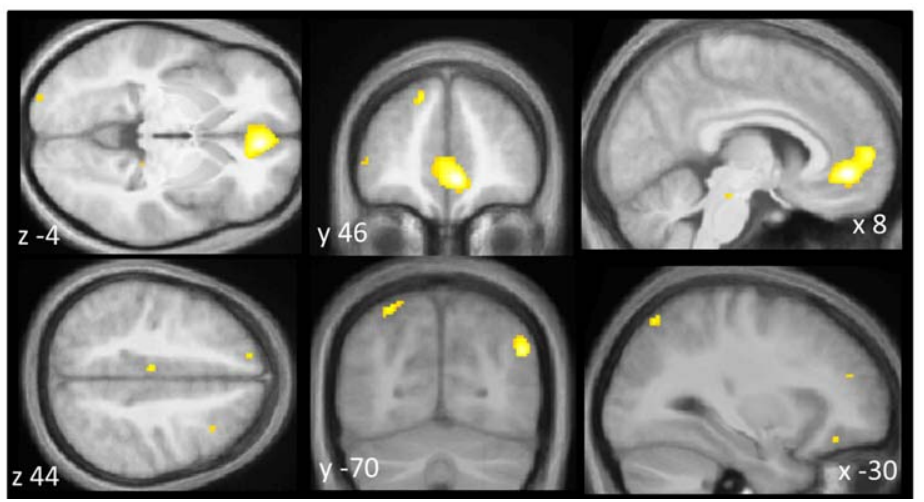

FIGURE 3: Sleep-loss-related time-on-task effects in the slowest RT domain and brain activity before stimulus appearance. (A1) Brain areas showing a significantly different time-on-task effect in the slowest RT domain between genotypes during sleep deprivation. (A2) Schematical display of the time course of thalamic brain activity [-8/-16/-4] over the task for a representative subject of each PER3 group. (B) Brain areas showing significantly more activation 2.2 seconds (equals 1 TR) before stimulus appearance with a subsequent slow response in $P E R 3^{55}$ than $P E R 3^{44}$ carriers during sleep deprivation. (A1) and (B): Overlay of statistical results on study population mean structural image, display at $p=0.001$ uncorrected. $R T=$ reaction time; TR $=$ time of repetition.

\section{KEY STRUCTURES OF THE DEFAULT MODE NET- WORK ARE MORE ACTIVATED BEFORE THE SLOW EVENTS UNDER SD IN THE VULNERABLE GENOTY-}

$P E$. Given that it has been shown previously that brain activity before stimulus appearance is predictive for subsequent lapses in attention, ${ }^{19}$ we analyzed brain activity immediately before stimulus appearance. Our results showed that immediately before slow RTs and compared to $P E R 3^{4 / 4}, P E R 3^{5 / 5}$ carriers had higher activation in regions commonly assigned to the default mode network $(\mathrm{DMN})^{30,38,39}$ (Table 5; Fig 4). No areas were more active in the PER3 $3^{4 / 4}$ compared to the PER3 $3^{5 / 5}$ (Table 5). Importantly, before fastest RTs, we did not observe any genotype-related differences (SD 55>44 n.s. at $p=0.001$ uncorrected level, data not shown). Please note that in this analysis, we did not test for functional connectivity of the DNM, but merely observed greater activity in regions that were assigned to this network in previous reports. ${ }^{30,38,39}$

\section{Discussion}

In the present study, we investigated cerebral correlates underlying performance during individually scheduled night-time (i.e., after 21 hours after wake-up time), when detrimental effects of sleep loss on cognition are strongest. For the first time, a SD and a multiple nap protocol was combined in order to quantify the impact of differential sleep pressure conditions at this critical circadian time window. By considering a polymorphism in the clock gene PER3, we stratified our group according to genetic susceptibility to total sleep loss. Performing the PVT, an attention-demanding task, ${ }^{25}$ more SD-vulnerable ${ }^{9,20}$ participants $\left(P E R 3^{5 / 5}\right)$ showed a consistent pattern of brain activity decreases under high sleep pressure, whereas in the more resilient participants $\left(P E R 3^{4 / 4}\right)$ brain activity increased. In addition, arousal-promoting areas were progressively less recruited with $\mathrm{To} T$ in vulnerable subjects and less functionally connected to other arousal- and attention-related brain structures under SD. Vulnerable participants were additionally more prone to activate structures associated with the default mode network when no stimulus was present.

Similar to earlier SD studies, ${ }^{15,28,40}$ we observed that $\mathrm{SD}$ during night-time is associated with increased activation in subcortical structures, whereas under low sleep pressure, several cortical regions were more active. 
TABLE 5. Differences in Brain Activity Before Slowest RTs During Sleep Deprivation According to Genotype

\begin{tabular}{|c|c|c|c|c|c|c|c|}
\hline Brain Area & Side & Z score & $p_{s v c}$ & $\mathbf{x}$ & $\mathbf{y}$ & $\mathbf{z}$ & $\operatorname{Re}$ \\
\hline \multicolumn{8}{|c|}{$\begin{array}{l}\text { Areas with greater activity one TR }\left(2.2 \text { seconds) before stimulus appearance in } P E R 3^{55} \text { than } P E R 3^{44} \text { ca }\right. \\
\text { (SD 55>44) }\end{array}$} \\
\hline Superior/middle frontal gyrus & $\mathrm{R}$ & 3.40 & 0.0003 & 32 & 22 & 46 & 19 \\
\hline Superior occipital gyrus & $\mathrm{L}$ & 3.18 & 0.0007 & -38 & -78 & 28 & 19 \\
\hline \multirow[t]{5}{*}{ Medial frontal gyrus } & $\mathrm{R}$ & 4.20 & 0.0000 & 8 & 46 & -4 & 31 \\
\hline & $\mathrm{R}$ & 3.48 & 0.0002 & 2 & 54 & 10 & 30 \\
\hline & $\mathrm{L}$ & 3.48 & 0.0002 & -2 & 56 & 10 & 30 \\
\hline & $\mathrm{L}$ & 3.48 & 0.0003 & -6 & 58 & 14 & 30 \\
\hline & $\mathrm{L}$ & 3.33 & 0.0004 & -2 & 50 & 8 & 30 \\
\hline Dorsal ACC & $\mathrm{R}$ & 4.26 & 0.0000 & 6 & 40 & -6 & 30 \\
\hline Dorsal ACC & $\mathrm{R}$ & 3.93 & 0.0000 & 2 & 42 & -8 & 30 \\
\hline Posterior/middle cingulate & $\mathrm{L}$ & 3.39 & 0.0004 & -6 & -20 & 44 & 32 \\
\hline Superior parietal gyrus & $\mathrm{R}$ & 3.93 & 0.0000 & 46 & -66 & 32 & 31 \\
\hline \multirow[t]{3}{*}{ Gyrus angularis/prencuneus } & $\mathrm{L}$ & 3.56 & 0.0002 & -28 & -70 & 56 & 14 \\
\hline & $\mathrm{L}$ & 3.29 & 0.0005 & -24 & -68 & 60 & 14 \\
\hline & $\mathrm{L}$ & 3.25 & 0.0006 & -20 & -66 & 60 & 14 \\
\hline \multirow[t]{2}{*}{ Medial temporal gyrus } & $\mathrm{L}$ & 3.70 & 0.0001 & -62 & -14 & -22 & 33 \\
\hline & $\mathrm{R}$ & 3.85 & 0.0001 & 56 & -6 & -16 & 33 \\
\hline
\end{tabular}

Areas with greater activity one TR (2.2 seconds) before stimulus appearance in $P E R 3^{44}$ than PER3 $3^{55}$ carriers (SD $55>44$ )

n.s. at $p=0.001$ uncorrected level

Coordinates $(\mathrm{x}, \mathrm{y}, \mathrm{z})$ are expressed in $\mathrm{mm}$ in the Montreal Neurological Institute (MNI) space. $p_{s v c}: p$ value after correction for multiple comparisons over small volumes of interest taken from the literature.

$\mathrm{TR}=$ time of repetition; $\mathrm{SD}=$ sleep deprivation; Ref. = references for coordinates; $\mathrm{R}=$ right; $\mathrm{L}=$ left; $\mathrm{B}=$ bilateral; $\mathrm{ACC}=$ anterior cingulate cortex.

Notably, many SD studies reported higher activations in frontoparietal areas when performance was preserved, and activity decreases when performance declined (reviewed in reference ${ }^{15}$ ). Although we observed significantly lower slowest RTs in the SD, these deactivations were located in the middle temporal gyrus, in the insula and the cuneus under high versus low sleep pressure when pooling both genotype groups. This discrepancy is likely due to the difference in circadian phase and duration of sleep loss, when compared to daytime SD studies, or alternatively, divergent task characteristics.

The consistent decreases in brain areas under high sleep pressure in the more vulnerable group $\left(P E R 3^{5 / 5}\right)$, and the increased brain activity in the more resilient group $\left(P E R 3^{4 / 4}\right)$ support data from Vandewalle et al., ${ }^{14}$ who investigated the impact of the PER3 genotype on cerebral correlates of working memory in the morning after SD. According to Drummond et al., ${ }^{18}$ activity increases underlying slow RTs after SD may reflect attentional recovery, given that compensatory behavior (e.g., anticipatory or fast responses) often follows slow RTs or lapses. ${ }^{4}$ In our data, a set of the brain regions showing higher activity in $P E R 3^{4 / 4}$ carriers during SD was positively associated with faster responses to the subsequent stimulus. This favors the assumption of compensatory recruitment, potentially leading to attentional recovery in the more resilient group. Similarly, Chee and $\operatorname{Tan}^{40}$ showed that in contrast to more resilient, the cognitively more vulnerable participants were unable to increase frontoparietal activation during a selective attention task under SD. The same authors showed that the ability to raise activation in response to lapses was lower under $\mathrm{SD}$, and during lapses, visual cortex and thalamic activation was reduced in comparison to lapses in well-rested conditions, which was interpreted as deactivations indicative for a loss of top-down control. ${ }^{41}$ Remarkably, we 
observed a similar pattern in the $P E R 3^{5 / 5}$ carriers, whereas the activity of our $P E R 3^{4 / 4}$ carriers better matched the well-rested pattern observed by Chee et al. In regard to sleepiness, a study by Czisch et al. ${ }^{42}$ showed compensatory activations within insular regions comparing a physiologically more alert to a more sleepy state (EEG-derived during task) after SD in an oddball task. This parallels our findings of the physiologically sleepier group $\left(P E R 3^{5 / 5}\right.$ carriers, see a previous work $\left.{ }^{9}\right)$, which also shows less task-related activation in an insular region. With respect to subjective sleepiness, our more SD-resilient participants increased activation in the left IFG, whereas the more vulnerable decreased activity in this region with increasing subjective sleepiness, which confirms previous reports of a positive relation of activation in this region with subjective sleepiness under SD. ${ }^{43,44}$

RTs did not significantly differ between genotypes in the slowest range, although $P E R 3^{5 / 5}$ carriers had significantly more lapses (RTs $>500 \mathrm{~ms}$ ) across the entire SD, particularly also with increasing ToT. ${ }^{9,20}$ Notably, genotypes did not differ in the slowest $25 \%$ of RTs with increasing ToT. However, on the cerebral level, the genotypes had different BOLD activity time courses, such that $P E R 3^{5 / 5}$ carriers decreased thalamic and ACC activity, supposedly mirroring a greater ToT-related vigilance decrement, whereas the PER3 $3^{4 / 4}$ carriers show relatively stable levels of activation within these regions. Similarly, Chee and $\operatorname{Tan}^{40}$ showed that vulnerable subjects suppress thalamic activity during lapses (defined as long RTs) after $\mathrm{SD}$, whereas more resilient ones tended to elevate. Thus, the $P E R 3^{5 / 5}$ genotype might be less able to sustain attention through "mental effort" mirrored on the cerebral level, ${ }^{45}$ potentially leading to a greater number of lapses. Notably, the thalamus has numerous projections to corti$\mathrm{cal}$ areas, but also receives input from ascending arousal systems, thereby mediating bottom-up arousal. ${ }^{46} \mathrm{We}$ observed increased functional connectivity between a part of the reticular formation (paramedian part of the midbrain) and a more superior brainstem area, the thalamus, the left IFG, and an occipital area in the resilient genotype. Importantly, activity in a brainstem region close to our seed region was shown to negatively correlate with slow wave sleep, ${ }^{36}$ further indicating that this area is potentially implicated in sleep-wake-related arousal promotion. This strengthens the plausibility that the differences between vulnerable and resilient genotypes result partly from a stronger ascending arousal promotion in PER3 $3^{4 / 4}$.

Considering the greater number of lapses and the higher amount of slow eye movements and sleep attacks in the more vulnerable $P E R 3^{5 / 5}$ genotype during $\mathrm{SD}$, it is tempting to interpret the failure to recruit "compensatory" brain areas during slowest RT as a greater tendency or even a precursor to drift into an "offmode" or default mode when no stimulus is present. This is supported by the previous finding that areas of the DMN are more activated before lapses. ${ }^{19}$ Our data are conceptually in line with this observation, given that immediately before the appearance of a stimulus, areas commonly assigned to the DMN (e.g., dorsal anterior cingulate cortex, posterior cingulate cortex, precuneus, and medial frontal areas) ${ }^{30,33,39}$ were more activated in the vulnerable $P E R 3^{5 / 5}$ carriers than in the resilient group under high sleep pressure. Interestingly, if taking into account the specific pattern during the slowest RT for each group separately, only PER $3^{5 / 5}$ carriers showed deactivations under $\mathrm{SD}$, mostly in areas compatible with the DMN. ${ }^{30,33,39}$ These participants are probably less able to sustain an active attentional network while they are waiting for stimuli, and thus switch into a more passive default mode, ${ }^{47}$ which, in turn, needs stronger suppression as soon as the stimulus appears. This may reflect a coping strategy of the more vulnerable participants leading to similar RTs for both genotypes at the behavioral level. However, because the PER $3^{5 / 5}$ carriers had more lapses, this coping process seems less successful or frailer to behavioral instability. These assumptions only account for nonoptimal performance, which is typically observed under SD and can be fatal, especially when appearing insidiously, eventually resulting in total lapses.

Finally, under low sleep pressure achieved by multiple naps, along with comparable vigilance levels and electrophysiological activity, ${ }^{9,20}$ brain activity was similar for both groups. Thus, the two genotypes did not differ substantially in their response to adverse circadian phase in the absence of high homeostatic sleep pressure levels. In this line, it is important to note that the $P E R 3^{5 / 5}$ carriers have a roughly $10 \%$ higher nap sleep efficiency over the entire circadian cycle. ${ }^{9}$ Although sleep efficiency is not a classical marker of sleep homeostasis, ${ }^{3}$ this finding suggests that between the naps, the homeostatic buildup may have been faster in the $P E R 3^{5 / 5}$ carriers, and that higher sleep efficiency might be necessary to reach equally low homeostatic levels compared to more resilient $P E R 3^{4 / 4}$. Homeostatic sleep pressure, operationalized here by a manipulation of the state (SD vs. NP), and presumably by trait $\left(P E R 3^{4 / 4}\right.$ vs. PER $\left.3^{5 / 5}\right),{ }^{12}$ seems thus to potentiate the harmful effects of adverse circadian phase at the cerebral level.

Our modest sample size presents a limitation in interpreting our results. Nevertheless, by selecting healthy participants without sleep complaints and controlling for confounding variables, we chose a homogenous phenotype 
across both genotypes. By implementing stringently controlled laboratory conditions, we minimized potential masking factors. The present analysis does not include a well-rested condition (daytime after regular sleep), restricting our findings to the present circadian window. However, the focus of this study was to compare the same circadian phase under differential sleep pressure conditions, and our results relate to being awake at night. Finally, fMRI is an indirect brain activity measure assessing relative changes in BOLD activity. We thus cannot rule out whether activity changes observed here result from changes in absolute brain activity. However, a recent study suggested that neural consequences of SD are mainly observed in signals evoked in task-relevant brain regions. ${ }^{48}$

By comparing two groups that were characterized with regard to vulnerability to SD with a multimethod approach (i.e., electrophysiology, subjective sleepiness, nap sleep, and vigilant attention ${ }^{9,20}$ ), we were able to infer patterns of resilience and vulnerability at the cerebral level. Our study revealed that the sensitivity to SD observed for tasks with low stimulus predictability might be based on a shift into the task-inactive DMN between relevant stimuli. Given that some people seem to have a greater predisposition to drift into these networks competing with goal-directed attention under SD, our findings might have implications to optimally adapt environment or instructions for night workers.

\section{Acknowledgment}

This work was supported by the Swiss National Science Foundation (\# 310030_130689) and by Förderbeitrag L. $\&$ Th. La Roche-Stiftung, and by the Niklaus and Bertha Burkhardt-Bürgin Stiftung (M.M.).

We thank Marie-France Dattler, Claudia Renz, Giovanni Balestrieri, Sarah Chellappa, Amandine Valomon, and all study helpers and interns for help in data acquisition; Hans-Peter Landolt, Valérie Bachmann, Andreas Papassotiropoulos, Dominique de Quervain, Kim Dung Huyn, Susanne Timus, Catherine Malgat, Sebastian Holst, Jurian Zürcher, Nikolaus Schäfer, and Wolfgang Berger for providing help with equipment; and Dr. med. Thomas Götz for medical screenings.

\section{Authorship}

M.M.: study design, data collection, analysis, and manuscript preparation; C.R.: study design, data collection, analysis, and manuscript preparation; V.G.: data collection and manuscript preparation; AV: manuscript preparation; C.P.: analysis and manuscript preparation; J.K.: data collection and manuscript preparation; K.S.: manu- script preparation; M.K.: manuscript preparation and study design; WS: manuscript preparation; C.C.: study design, data collection, analysis, and manuscript preparation; CS: study design, data collection, analysis, and manuscript preparation.

\section{Potential Conflicts of Interest}

Nothing to report.

\section{References}

1. Wright $\mathrm{KP}$, Lowry CA, Lebourgeois MK. Circadian and wakefulness-sleep modulation of cognition in humans. Front Mol Neurosci 2012;5:50.

2. Dijk DJ, Czeisler CA. Paradoxical timing of the circadian rhythm of sleep propensity serves to consolidate sleep and wakefulness in humans. Neurosci Lett 1994;166:63-68.

3. Borbely AA. A two process model of sleep regulation. Hum Neurobiol 1982;1:195-204.

4. Doran SM, Van Dongen HP, Dinges DF. Sustained attention performance during sleep deprivation: evidence of state instability. Arch Ital Biol 2001;139:253-267.

5. Lim J, Dinges DF. Sleep deprivation and vigilant attention. Ann N Y Acad Sci 2008;1129:305-322.

6. Graw P, Krauchi K, Knoblauch V, et al. Circadian and wakedependent modulation of fastest and slowest reaction times during the psychomotor vigilance task. Physiol Behav 2004;80: 695-701.

7. Van Dongen HP, Baynard MD, Maislin G, Dinges DF. Systematic interindividual differences in neurobehavioral impairment from sleep loss: evidence of trait-like differential vulnerability. Sleep 2004;27:423-433.

8. Lo JC, Groeger JA, Santhi N, et al. Effects of partial and acute total sleep deprivation on performance across cognitive domains, individuals and circadian phase. PLoS ONE 2012;7:e45987.

9. Maire M, Reichert CF, Gabel V, et al. Sleep ability mediates individual differences in the vulnerability to sleep loss: evidence from a PER3 polymorphism. Cortex 2014;52:47-59.

10. Groeger JA, Viola AU, Lo JC, et al. Early morning executive functioning during sleep deprivation is compromised by a PERIOD3 polymorphism. Sleep 2008;31:1159-1167.

11. Viola AU, Archer SN, James LM, et al. PER3 polymorphism predicts sleep structure and waking performance. Curr Biol 2007; 17:613-618.

12. Dijk DJ, Archer SN. PERIOD3, circadian phenotypes, and sleep homeostasis. Sleep Med Rev 2010;14:151-160.

13. Goel N, Banks S, Mignot E, Dinges DF. PER3 polymorphism predicts cumulative sleep homeostatic but not neurobehavioral changes to chronic partial sleep deprivation. PLoS ONE 2009;4: e5874.

14. Vandewalle G, Archer SN, Wuillaume C, et al. Functional magnetic resonance imaging-assessed brain responses during an executive task depend on interaction of sleep homeostasis, circadian phase, and PER3 genotype. J Neurosci 2009;29:79487956.

15. Chee MW, Asplund CL. Neuroimaging of attention and alterations of processing capacity in sleep-deprived persons. In Nofzinger EA, Maquet P, Thorpy MJ, eds. Neuroimaging of Sleep and Sleep Disorders. Cambridge, UK: Cambridge University Press, 2013:137-144. 
16. Dorrian J, Rogers N, Dinges D. Psychomotor vigilance performance: a neurocognitive assay sensitive to sleep loss. In: Kushida C, ed. Sleep Deprivation: Clinical Issues, Pharmacology، and Sleep Loss Effects. New York: Marcel Dekker, 2005:39-70.

17. Cajochen C, Knoblauch V, Krauchi K, et al. Dynamics of frontal EEG activity, sleepiness and body temperature under high and low sleep pressure. Neuroreport 2001;12(10):2277-81.

18. Drummond SP, Bischoff-Grethe A, Dinges DF, et al. The neural basis of the psychomotor vigilance task. Sleep 2005;28:10591068

19. Weissman DH, Roberts KC, Visscher KM, Woldorff MG. The neural bases of momentary lapses in attention. Nat Neurosci 2006;9:971-978.

20. Maire M, Reichert CF, Gabel V, et al. Time-on-task decrement in vigilance is modulated by inter-individual vulnerability to homeostatic sleep pressure manipulation. Front Behav Neurosci 2014;8:59.

21. Knoblauch V, Krauchi K, Renz C, et al. Homeostatic control of slow-wave and spindle frequency activity during human sleep: effect of differential sleep pressure and brain topography. Cereb Cortex 2002;12:1092-1100.

22. Dinges DF, Powell JW. Microcomputer analyses of performance on a portable, simple visual RT task during sustained operations. Behav Res Methods Instrum. Comput 1985;17:625-655.

23. Akerstedt T, Gillberg M. Subjective and objective sleepiness in the active individual. Int J Neurosci 1990;52:29-37.

24. Kenward MG, Roger JH. Small sample inference for fixed effects from restricted maximum likelihood. Biometrics 1997;53:983-997.

25. Basner M, Dinges DF. Maximizing sensitivity of the psychomotor vigilance test (PVT) to sleep loss. Sleep 2011;34:581-591.

26. Schmidt C, Collette F, Leclercq Y, et al. Homeostatic sleep pressure and responses to sustained attention in the suprachiasmatic area. Science 2009;324:516-519

27. Muto V, Shaffii-le Bourdiec A, Matarazzo L, et al. Influence of acute sleep loss on the neural correlates of alerting, orientating and executive attention components. J Sleep Res 2012;21:648658.

28. Tomasi $\mathrm{D}$, Wang RL, Telang $\mathrm{F}$, et al. Impairment of attentional networks after 1 night of sleep deprivation. Cereb Cortex 2009; 19:233-240.

29. Thomas $M$, Sing $H$, Belenky G, et al. Neural basis of alertness and cognitive performance impairments during sleepiness. I. Effects of $24 \mathrm{~h}$ of sleep deprivation on waking human regional brain activity. J Sleep Res 2000;9:335-352.

30. Laird AR, Eickhoff SB, Li K, et al. Investigating the functional heterogeneity of the default mode network using coordinatebased meta-analytic modeling. J Neurosci 2009;29:14496-14505.

31. Gujar N, Yoo SS, Hu P, Walker MP. The unrested resting brain: sleep deprivation alters activity within the default-mode network. J Cogn Neurosci 2010;22:1637-1648.

32. Leech R, Kamourieh S, Beckmann CF, Sharp DJ. Fractionating the default mode network: distinct contributions of the ventral and dorsal posterior cingulate cortex to cognitive control. J Neurosci 2011;31:3217-3224.

33. Fransson $\mathrm{P}$, Marrelec $\mathrm{G}$. The precuneus/posterior cingulate cortex plays a pivotal role in the default mode network: evidence from a partial correlation network analysis. Neuroimage 2008;42:11781184

34. Gitelman DR, Penny WD, Ashburner J, Friston KJ. Modeling regional and psychophysiologic interactions in fMRI: the importance of hemodynamic deconvolution. Neuroimage 2003; 19:200-207.

35. O'Reilly JX, Woolrich MW, Behrens TE, et al. Tools of the trade: psychophysiological interactions and functional connectivity. Soc Cogn Affect Neurosci 2012;7:604-609

36. Maquet $P$, Degueldre C, Delfiore $G$, et al. Functional neuroanatomy of human slow wave sleep. J Neurosci 1997;17: 2807-2812.

37. Henson R, Friston K. Convolution models for fMRI. In: Penny WD Friston KJ, Ashburner JT, eds. Statistical Parametrical Mapping: The Analysis of Functional Brain Images, 1st ed. New York: Academic, 2007:178-192.

38. Mantini D, Vanduffel W. Emerging roles of the brain's default network. Neuroscientist 2013;19:76-87.

39. Buckner RL, Andrews-Hanna JR, Schacter DL. The brain's default network: anatomy, function, and relevance to disease. Ann N Y Acad Sci 2008;1124:1-38.

40. Chee MW, Tan JC. Lapsing when sleep deprived: neura activation characteristics of resistant and vulnerable individuals. Neuroimage 2010;51:835-843.

41. Chee MW, Tan JC, Zheng $H$, et al. Lapsing during sleep deprivation is associated with distributed changes in brain activation. J Neurosci 2008;28:5519-5528.

42. Czisch M, Wehrle R, Harsay HA, et al. On the need of objective vigilance monitoring: effects of sleep loss on target detection and task-negative activity using combined EEG/fMRI. Front Neurol 2012;3:67.

43. Drummond SP, Gillin JC, Brown GG. Increased cerebral response during a divided attention task following sleep deprivation. J Sleep Res 2001;10:85-92.

44. Drummond SP, Brown GG, Gillin JC, et al. Altered brain response to verbal learning following sleep deprivation. Nature 2000;403 655-657.

45. Portas CM, Rees G, Howseman AM, et al. A specific role for the thalamus in mediating the interaction of attention and arousal in humans. J Neurosci 1998;18:8979-8989.

46. Steriade M, Llinas RR. The functional states of the thalamus and the associated neuronal interplay. Physiol Rev 1988;68:649-742.

47. Sonuga-Barke EJ, Castellanos FX. Spontaneous attentional fluctuations in impaired states and pathological conditions: a neurobiological hypothesis. Neurosci Biobehav Rev 2007;31:977986.

48. Asplund $\mathrm{CL}$, Chee MW. Time-on-task and sleep deprivation effects are evidenced in overlapping brain areas. Neuroimage 2013;82:326-335.

49. Buysse DJ, Reynolds CF 3rd, Monk TH, et al. The Pittsburgh Sleep Quality Index: a new instrument for psychiatric practice and research. Psychiatry Res 1989;28:193-213.

50. Johns MW. A new method for measuring daytime sleepiness: the Epworth sleepiness scale. Sleep 1991;14:540-545.

51. Horne JA, Östberg O. A self-assessment questionnaire to determine morningness-eveningness in human circadian rhythms. Int $J$ Chronobiol 1976;4:97-110.

52. Roenneberg T, Wirz-Justice A, Merrow M. Life between clocks: daily temporal patterns of human chronotypes. J Biol Rhythms 2003;18:80-90.

53. Beck AT, Steer RA, Brown GK. BDI-II, Beck Depression Inventory: Manual, 2nd ed. Boston, MA: Harcourt Brace, 1996. 